\title{
A Chinese family affected by lynch syndrome caused by MLH1 mutation
}

\author{
Shuqin Jia ${ }^{1+}$, Meng Zhang ${ }^{1+}{ }^{1}$, Yu Sun ${ }^{2}$, Hai Yan ${ }^{1,3}$, Fangping Zhao ${ }^{4}$, Ziyu Li $5^{5^{*}}$ and Jiafu $\mathrm{Ji}^{1,5^{*}}$
}

\begin{abstract}
Background: Lynch syndrome (LS) is caused by mutations in DNA mismatch repair (MMR) genes, which accounts for 3-5\% of colorectal cancer. The risks of several types of cancer are greatly increased among individuals with LS. In this study, 4 members of a Chinese family with a MLH1 pathogenic variant, resulting in colonic carcinoma, was reported.

Case presentation: A 52-year-old colon cancer female was brought to us with a family history of colon cancer. Genetic counseling traced 4 members in her family with colon cancer (mother and 3 siblings including the proband) as well as other cancer types. Next generation sequencing (NGS) with a multiple gene panel including MMR genes showed a germline mutation in MLH1 (c.1852_1854delAAG, p.K618del) in all 3 affected family members and confirmed the diagnosis of Lynch syndrome. In addition, this mutation was also identified in a asymptomatic offspring, who was then recommended to a prophylactic measure against cancer. A personalized health care plan was implemented for monitoring the condition and progression of the affected individuals.

Conclusion: Based on public database searching followed by pedigree verification, p.K618del variant in MLH1 is a pathogenic mutation, which supported the diagnosis of LS. This case highlights the importance of diagnosis and management in patients with hereditary cancer syndromes, particularly for asymptomatic family members.
\end{abstract}

Keywords: Lynch syndrome, Hereditary, Colorectal carcinoma, Genetic counseling, Case report

\section{Background}

Approximately 1.4 million new colorectal cancer (CRC) cases occurred in 2012 worldwide [1]. The majority of CRCs are sporadic which is caused by comprehensive genetic and environmental factors, whereas, hereditary CRCs account for $5-10 \%$ of total CRCs. Moreover, an approximately 3 to $5 \%$ of CRCs are caused by Lynch syndrome (LS) which is also called hereditary non-polyposis colorectal cancer (HNPCC) $[2,3]$. Patients with LS have a $52-82 \%$ lifetime risk of colorectal carcinoma and $6-13 \%$ risk of gastric cancer, as well as increased risks of developing other

\footnotetext{
* Correspondence: ligregory369@hotmail.com; jijiafu@hsc.pku.edu.cn Shuqin Jia and Meng Zhang contributed equally to this work. ${ }^{\dagger}$ Equal contributors

${ }^{5}$ Department of Gastrointestinal Surgery, Key laboratory of Carcinogenesis and Translational Research (Ministry of Education), Peking University Cancer Hospital \& Institute, No.52 Fucheng Road, Haidian District, Beijing 100142, China

${ }^{1}$ Center for Molecular Diagnostics, Key laboratory of Carcinogenesis and Translational Research (Ministry of Education), Peking University Cancer Hospital \& Institute, No.52 Fucheng Road, Haidian District, Beijing 100142, China

Full list of author information is available at the end of the article
}

cancer types including small bowel, liver, gallbladder ducts, urinary tract, pancreas, brain and skin. In females, LS confers increased risks of both endometrial (25-60\%) and ovarian cancers (4-12\%) [4]. LS cancer risk is inherited in an autosomal dominant pattern, and is caused by germline variations in DNA mismatch repair (MMR) genes such as MLH1, MSH2, MSH6, or PMS2. Approximately 90\% of LS patients could potentially carry variations in $M L H 1$ and MSH2, 7\% carry mutations in MSH6, and less than 5\% are likely to have PMS2 mutations [4]. The normal function of the MMR proteins is to correct errors during DNA synthesis and prevent recombination between non-identical sequences. Mutations in any of these genes may lead to genomic instability, manifesting microsatellite instability (MSI) [5]. These gene variations could be identified by sequencing methods. Since the breakthrough of the next-generation sequencing (NGS) technology, the scale of genomic information acquired by such manner is significantly increased while the cost is dramatically reduced. Its core idea is sequencing by synthesis, which is to identify the DNA sequencing by capturing the signal as a nucleotide

(c) The Author(s). 2018 Open Access This article is distributed under the terms of the Creative Commons Attribution 4.0 International License (http://creativecommons.org/licenses/by/4.0/), which permits unrestricted use, distribution, and 
added to the growing strand. Microsatellites are short repetitive DNA sequences distributed throughout human genome. They are widely used for DNA profiling in cancer diagnosis. Increases or decreases in repeat length within the microsatellites markers, detected from the test DNA sample and normalized by matching normal, were analyzed by capillary electrophoresis. Alleles that are presented in the test DNA sample but not found in the corresponding normal samples indicate MSI. The development of these technologies enables the diagnosis of hereditary cancer syndrome more efficient and accurate than ever before, providing clinicians with quick guidance on the subsequent health care management strategies.

Cancer genetic counseling is essential for the health management of patients and their families with hereditary cancer syndromes. However, there is still a lack of standards in clinical practice in China. In this study, we report a case of a Chinese family with LS, and how we make the diagnosis and a implementation of a personalized health management plan using modern clinical molecular techniques and professional genetic counseling.

\section{Case presentation}

The patient, a 52-year-old female, was admitted to the department of gastrointestinal surgery of Peking University Cancer Hospital \& Institute in September, 2016, due to space-occupying lesions in the colon found by colonoscopy during medical examination 2 weeks before. Histopathological examination of endoscopic biopsy specimens indicated moderately differentiated colonic adenocarcinoma. Contrast-enhanced computer tomography (CT) of her abdomen demonstrated that the intestinal wall was thickened about $14 \mathrm{~mm}$ in the transverse colon, and several small lymph nodes $(7 \mathrm{~mm})$ scattered around the intestine were detected (Fig. 2a and b). Laboratory examination revealed that the levels of CEA and CA72.4 increased to $15.17 \mathrm{ng} / \mathrm{ml}$ and $20.88 \mathrm{U} / \mathrm{ml}$ respectively. Laparoscopic examination confirmed the tumor $(6 \mathrm{~cm} \times 5 \mathrm{~cm})$ was located in the hepatic flexure of the colon (Fig. 2c and d).

The patient (III7) had no other major medical history, except a family history of colon cancer in 3 out of 5 first-degree relatives (mother II2; sister, III5; brother, III6). In particular, the patient's mother (II2) was diagnosed two separate primary colon cancer at the age 54 and 61 at different sites. Her sister (III5) was diagnosed with endometrial cancer and colon cancer at the age of 54 and 61 respectively (Fig. 1). The patient was referred to our cancer genetic counseling clinic for LS genetic testing. Based on Amsterdam II criteria, the proband was diagnosed with LS.

To confirm the diagnosis, all affected individuals (III5, 6 and 7) underwent genetic testing of a 101-gene panel by next generation sequencing. Peripheral blood was collected to extract genomic DNA (gDNA). The gDNA was then used to generate libraries according to the protocols suggested by Illumina. A custom targeted capture kit, covering all exons of the 101 genes, was designed (Agilent Technologies, Additional file 1: Table S1) [6]. Qualified libraries were subsequently sequenced on the Illumina HiSeq 2500 platform with $2 \times 150$ bp configuration. Reads were aligned to the reference human genome GRCh37 with BWA and PCR duplications were marked using Picard tools (version 1.57). To further

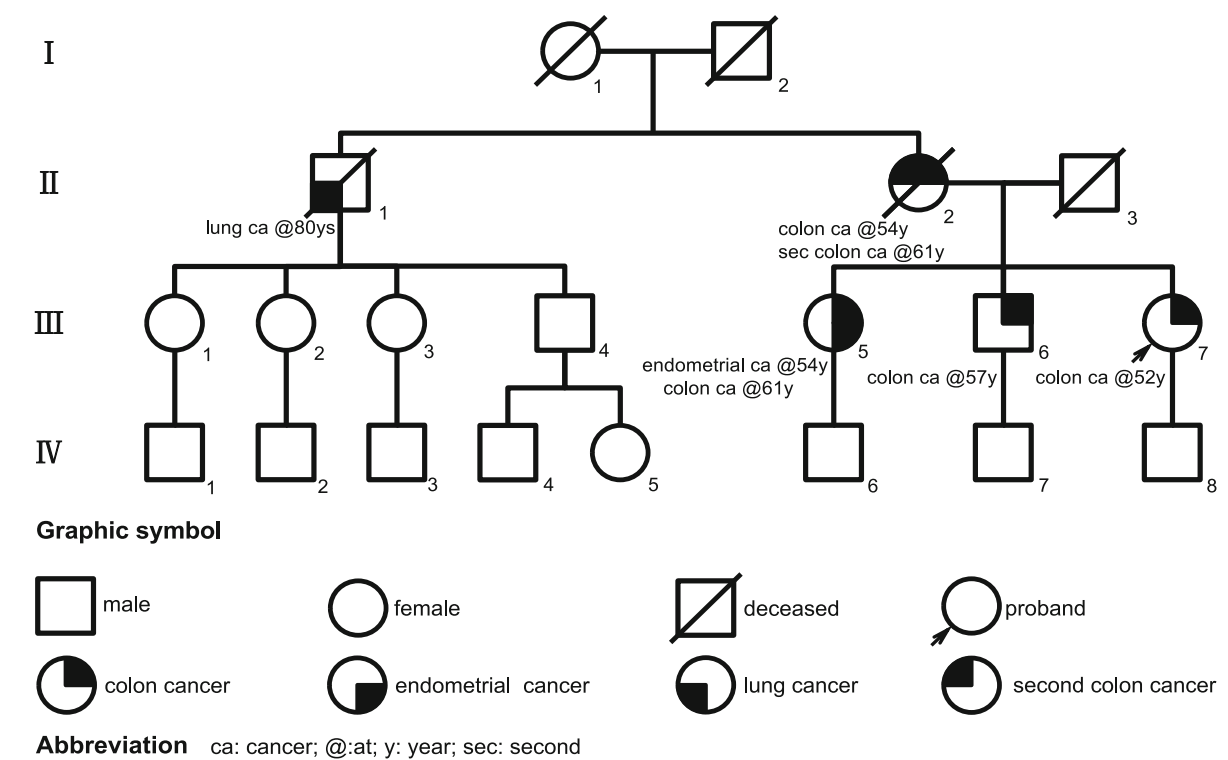

Fig. 1 Pedigree. Affected individuals III5, III6 and III7(proband) all had colon cancer and all carry the same MLH1 mutation (Table 1). With the history of colon cancer, II2 is predicted to the carrier of MLH1 mutation in the family. Among offspring of the affected individuals, IV8 carries the same MLH1 mutation (Table 1) and underwent preventative therapy 
increase the specificity for mutation calling, realignment and base recalibration were conducted using Genome Analysis tool kit (GATK). All samples were tested at least in an average depth of 200 -fold coverage. Bases with a minimum of 30-fold coverage was required at every targeted position (Additional file 2: Table S2). The missense, nonsense, indel and splice site mutations that located at the upstream or downstream 1-2 bp of exon, whose frequency are below $5 \%$ in at least one pubic population database were retained (Additional file 3: Table S3). According to the American College of Medical Genetics (ACMG) standards and guidelines for the interpretation of sequence variants, all the gene variants were classified into 5 grades. Therefore, 14 mutations at least carried by two first-degree relatives were listed. A pathogenic variant (class 5) in MLH1 (c.1852_1854delAAG, p.K618del) was identified in all patient's blood samples (Table 1).

Laparoscopy-assisted colectomy was performed on the proband to resect right colonic mass and its surrounding tissue followed by ileocolonic anastomosis. In addition, the clinicopathologic stage was PT3NOM0 and no complications occurred in the perioperative period. Conventional hematoxylin and eosin staining and immunohistochemistry were performed on resected specimens to confirm the malignancy (Fig. 2e and f). Additionally, abdominal CT of III5 showed a obstructing mass in the same location of colon as the proband (III7) (Fig. $2 \mathrm{~g}$ and h). Moreover, according to the medical record for the proband's brother (III6) in another hospital, a tumor was found in his hepatic flexure of colon. Immunohistochemistry results showed MMR deficiency in all tumor tissues of the 3 cancer patients (Table 2). Subsequently, MSI testing was performed using MSI Analysis System Version 1.2 (Promega). Tumor DNA was extract from formalin fixed paraffin embedded sections. Genomic DNA extracted from white blood cell was used as normal control. Seven markers were amplified using fluorescent PCR. The PCR products were separated by capillary electrophoresis using Applied Biosystem 3130 Genetic Analyzer. GeneMapper Analysis Software was used to analyze the output data. The MSI results indicated that the 3 siblings with cancer (III5, 6 and 7) were all microsatellite instability-high (MSI-H) (Table 2). Based on the results of MSI and the proband's clinical stage, no adjuvant chemotherapy was given after surgery.

To screen and evaluate the cancer developing risk in the offspring, children of affected individuals were also enrolled for genetic testing. And 1 (IV8) out of 3 carries the same MLH1 mutation to the proband. However, this carrier has no symptoms or confirmed diagnosis of cancer. Endoscopy was performed on IV8 for further examination and the ileocecal mucosa showed signs of dysplasia, including chronic inflammation with erosion, lymphoid hyperplasia and mild atypical hyperplasia of glandular epithelium (Fig. 2i). The asymptomatic individual was given oral administration of aspirin as a preventative treatment, and 6-months follow-up showed improved appearance with colonoscopy examination (Fig. 2j). A healthcare plan was proposed to this offspring including colonoscopy and urine test once a year, and gastroscopy every $3 \sim 5$ years after 35 years old. All individuals carrying the $M L H 1$ mutation in this family will be monitored on a long term basis.

The CARE guidelines were followed in reporting this case.

Table 1 Sequencing result on genes associated with hereditary cancer syndrome in family members

\begin{tabular}{|c|c|c|c|c|c|c|c|c|c|}
\hline \multirow[t]{2}{*}{ Gene } & \multirow[t]{2}{*}{ cDNA change } & \multirow[t]{2}{*}{ AA change } & \multicolumn{6}{|c|}{ Patient } & \multirow{2}{*}{$\begin{array}{l}\text { Clinical } \\
\text { significance }\end{array}$} \\
\hline & & & 1115 & III6 & 1117 & IV 6 & IV 7 & IV 8 & \\
\hline EPCAM & c. $298 \mathrm{G}>\mathrm{A}$ & p.D100N & het & wt & het & het & wt & het & Likely benign \\
\hline FANCE & c. $1028 \mathrm{G}>\mathrm{A}$ & p.R343Q & het & het & wt & het & het & wt & Benign \\
\hline$M C 1 R$ & c. $488 \mathrm{G}>\mathrm{A}$ & p.R163Q & het & wt & het & wt & het & hom & Benign \\
\hline MLH1 & c.1852_1854delAAG & p.K618del & het & het & het & wt & wt & het & Pathogenic \\
\hline MLH1 & c. $655 A>G$ & p.1219V & het & het & het & het & wt & wt & Benign \\
\hline$N B N$ & C. $1690 G>A$ & p.E564K & het & het & het & wt & wt & het & Benign \\
\hline PALLD & c. 2069 C > A & p.P690H & wt & het & wt & wt & het & wt & Benign \\
\hline PMS2 & c.379G $>A$ & p.A127T & wt & het & het & wt & het & wt & Likely benign \\
\hline PTCH1 & c. $3583 \mathrm{~A}>\mathrm{T}$ & p.T1195s & het & het & het & wt & het & wt & Benign \\
\hline RHBDF2 & c. $28 \mathrm{~A}>\mathrm{C}$ & p.S10R & het & het & het & wt & het & wt & Benign \\
\hline SDHA & c. $113 \mathrm{~A}>\mathrm{T}$ & p.D38V & wt & wt & het & wt & $w t$ & het & Likely benign \\
\hline SLX4 & c. $1231 C>T$ & p.R411W & het & het & het & het & het & wt & Likely benign \\
\hline TSC2 & c. $856 \mathrm{~A}>\mathrm{G}$ & p.M286 V & het & het & het & wt & wt & het & Benign \\
\hline TSC2 & C.500G $>\mathrm{T}$ & p.W167 L & het & het & het & wt & wt & het & VUS \\
\hline
\end{tabular}

Next generation sequencing was performed using 101-gene panel developed by Genetron Health (Beijing) Co., Ltd 


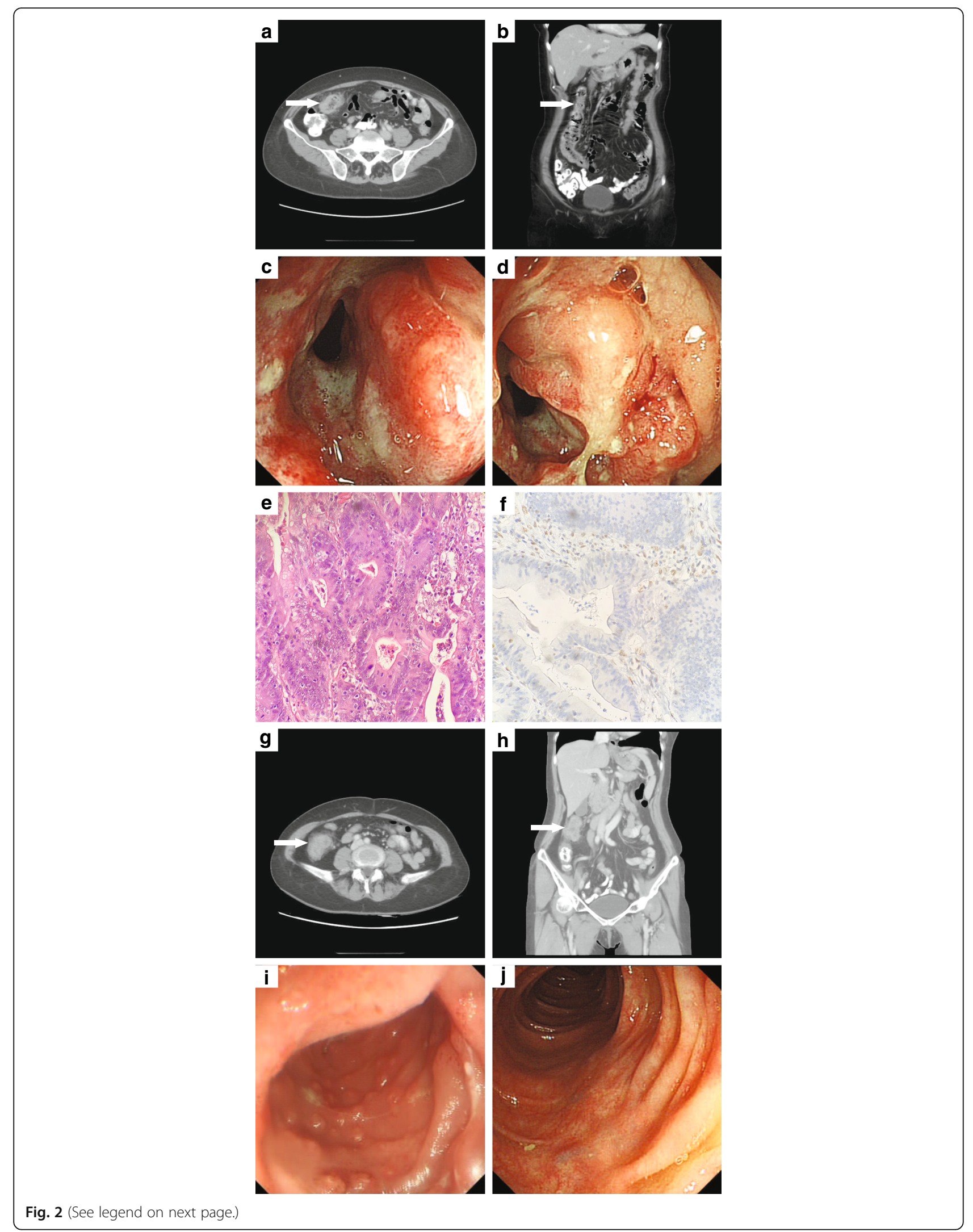


(See figure on previous page.)

Fig. 2 Clinical examination results. a and $\mathbf{b}$ Abdominal computed tomography (CT) scan of the proband (III7) revealed an obstructing mass located in the hepatic flexure of the colon (arrow). a, axial and b, coronal. c and $\mathbf{d}$ A space-occupying lesion in colon by colonoscopy in III7. e HE staining demonstrates moderately-differentiated colon adenocarcinoma with glandular architecture, nuclear atypia and dysregulated cell proliferation. $\mathbf{f}$ immunochemistry stain of MLH1. $\mathbf{g}$ and $\mathbf{h}$ Abdominal computer tomography of III5 showed a obstructing mass in the same location of colon as the proband (III7) (arrow). $\mathbf{g}$, axial view. h, coronal view. $\mathbf{i}$ and $\mathbf{j}$ Colonoscopy of IV8. i Before the preventative treatment, ileocecal mucosa showed sign of chronic inflammation with erosion, lymphoid hyperplasia and mild atypical hyperplasia of glandular epithelium. j After oral administration of aspirin for 6 months, same site of ileocecal mucosa showed improved appearance overall

\section{Discussion and conclusions}

CRC is the fifth diagnosed cancer in China [7]. The estimated numbers of CRC related new cases and deaths in China in 2015 were about 376,300 and 191,000 respectively. Among these patients, $75 \%$ was sporadic, whereas $25 \%$ represented a family history of cancer. Additionally, inherited colorectal cancer accounts for $5-10 \%$ of total CRCs, including LS, familial adenomatous polyposis, MUTYH-associated polyposis, Li-Fraumeni syndrome, Peutz-Jeghers syndrome and Juvenile polyposis syndrome [8]. LS is considered as an assemblage of related cancers characterized by defects in DNA MMR genes including MLH1, MSH2, MSH6, and PMS2. It is estimated that $90 \%$ of LS related CRCs and less than $10 \%$ of sporadic CRCs show MSI. LS confers a 52-82\% lifetime risk of CRCs. Individuals carrying $M L H 1$ or $M S H 2$ pathogenic variations appear to have the highest risk developing CRC, which occurs at the age of 44 on average.

In this case, a typical Chinese LS family was reported. With thorough genetic counseling prior to genetic testing, a clear LS history was established: the proband's mother (II2) suffered from second primary colon cancer, and her sister (III5) was also affected by endometrial cancer. Although the family history indicated the clinical diagnosis of LS, we could not make a definite diagnosis of LS without genetic testing. Therefore, a 101-gene panel that covered 65 common hereditary cancer syndromes was used. From clinical perspective, a single MMR gene testing once at a time using Sanger sequencing is more likely to be appropriate for the proband and her relatives. However, in this study, the proband was considered as LS based on family history without further test prior to the genetic test. The results of IHC and MSI were obtained after the surgery when the diagnosis was confirmed. Considering the comparable cost of Sanger sequencing all exons of different MMR genes and the NGS sequencing, we preferred NGS for its faster turnaround time. Besides, we would like to explore if there is any other potential colorectal cancer predisposing genes in Chinese population. Genetic testing reveals all 3 affected siblings carrying a pathogenic variant in $M L H 1$ (c.1852_1854delAAG). In addition, their tumors developed in the common location in colon. Further studies may help understand the pathological impact of this mutation during tumorigenesis.

Meanwhile, all offspring at risk of developing cancer were recommended for genetic testing and results showed that the proband's son (IV8) inherited the pathogenic variation. A chemoprevention strategy of aspirin oral administration was prescribed based on preclinical studies and clinical trials [9-11]. Furthermore, a personalized healthcare management plan was recommended to the young carrier for regular surveillance. As of this

Table 2 Detection of microsatellite instability

\begin{tabular}{|c|c|c|c|c|}
\hline Patient & & Proband III7 & Proband's Sister III5 & Proband's Brother III6 \\
\hline MSI status & & MSI-H & MSI-H & MSI-H \\
\hline \multirow[t]{5}{*}{ Genetic testing for microsatellite instability } & BAT-25 & MSI & MSI & MSI \\
\hline & BAT-26 & MSS & MSI & MSI \\
\hline & NR-21 & MSI & MSI & MSI \\
\hline & NR-24 & MSI & MSI & MSI \\
\hline & Mono-27 & MSS & MSI & MSI \\
\hline \multirow[t]{4}{*}{ Immunohistochemistry of MMR proteins } & MLH1 & - & - & - \\
\hline & MSH2 & + & + & - \\
\hline & MSH6 & + & + & - \\
\hline & PMS2 & - & - & + \\
\hline
\end{tabular}

Microsatellite instability was accessed at both protein and genetic levels. Immunohistochemistry of MMR proteins (MLH1, MSH2, MSH6 and PMS2) was performed to demonstrate the deficiency of MMR system in affected individuals. "-" suggests the deficiency of corresponding proteins and " + " suggests a normal expression of corresponding proteins. Deficiency in MMRs results in MSI

Genetic screening for MSI was performed using the BAT-25, BAT-26, NR-21, NR-25, and Mono-27 markers. MSS microsatellite stable, MSI microsatellite instability, when none of the five markers showed instability. MSI-H MSI-high, when two or more of the five markers showed instability. MSI-L MSI-low, when only one of the five markers showed instability 
study submission, signs of colonic dysplasia were under control after 6 months of aspirin treatment.

MSI-H caused by germline mutations in MLH1 is not only a key component of the pathogenesis of CRC but also act as a positive prognostic maker. Moreover, it can be used to predict the efficacy of chemotherapy [12] and immunotherapy [13]. All affected cancer patients in the family that were diagnosed with cancer underwent surgery without adjuvant therapies based on NCCN (V2.2016) guidelines [14]. In the case of the proband (III7), the latest CT scan was performed 16 months after the surgery and did not show any sign of cancer recurrence, and the surveillance and follow-up of this LS family is still ongoing.

The standard procedure of cancer genetic counseling includes pre-test counseling, result disclosure and follow-up. The purpose of genetic counseling is to help at-risk individuals and their families to understand the risks of cancers, to disclose the genetic test results, to discuss options for risk management and family planning, and to provide psychosocial support for individuals as needed. Cancer genetic counseling is just sprouting in China and will be facing many challenges such as shortage of qualified genetic counselors. This is expected to be solved with a better understanding of cancer genetics in both cancer patients and medical practitioners as well as better preventative education to the general population.

\section{Additional files}

Additional file 1: Table S1. 101 gene panel. The gene list of targeted capture panel used in the case. (XLSX $11 \mathrm{~kb}$ )

Additional file 2: Table S2. All six family members gene test result. All six family members gene test results displayed in order of gene. (XLSX $770 \mathrm{~kb}$ )

Additional file 3: Table S3. Individual sequencing result. All six family members gene test results displayed in individual form. (XLSX $32 \mathrm{~kb}$ )

\section{Abbreviations}

ACMG: American College of Medical Genetics; CRC: Colorectal cancer; CT: Computer tomography; GATK: Genome analysis tool kit; gDNA: Genomic DNA; HNPCC: Hereditary non-polyposis colorectal cancer; LS: Lynch syndrome; MMR: DNA mismatch repair; MSI: Microsatellite instability; MSI$\mathrm{H}$ : Microsatellite instability-high; NGS: Next-generation sequencing

\section{Acknowledgements}

We thank all family members for their participation. We would like to thank Dr. Lei Tang (Department of Radiology, Peking University Cancer Hospital \& Institute) for his valuable advice in interpreting computer tomography images.

\section{Funding}

This work was supported by funding from Hospital Program for Youth of Beijing Municipal Administration (QML20151003), Clinical Medicine Development Special Funding of Beijing Municipal Administration (ZYLX201701), and National Science and Technology Ministry (2014BAI09B02).

\section{Availability of data and materials}

The datasets during and/or analyzed during the current study available from the corresponding author on reasonable request.

\section{Authors' contributions}

$\mathrm{ZL}$ and JJ were involved in all aspects of patient care. MZ and SJ conducted genetic counseling, literature review and drafted the manuscript. YS, FZ and HY conducted genetic testing. ZL and JJ critically reviewed the manuscript. All authors approved the final manuscript.

\section{Ethics approval and consent to participate}

Reports describing the case have been performed in accordance with the Declaration of Helsinki and have been approved by the BEIJING CANCER HOSPITAL MEDICAL ETHICS COMMITTEE.

Written Informed consent to participate in the study was obtained from participants. A copy of the consent form is available.

\section{Consent for publication}

Consent to publish data, images or videos was obtained from any subject. A copy of the written consent is available for review by the Editor of this journal.

\section{Competing interests}

The authors declare that they have no competing interests.

\section{Publisher's Note}

Springer Nature remains neutral with regard to jurisdictional claims in published maps and institutional affiliations.

\section{Author details}

${ }^{1}$ Center for Molecular Diagnostics, Key laboratory of Carcinogenesis and Translational Research (Ministry of Education), Peking University Cancer Hospital \& Institute, No.52 Fucheng Road, Haidian District, Beijing 100142, China. ${ }^{2}$ Department of Pathology, Key laboratory of Carcinogenesis and Translational Research (Ministry of Education), Peking University Cancer Hospital \& Institute, Beijing, China. ${ }^{3}$ Department of Pathology, Duke University Medical Center, Durham, NC, USA. ${ }^{4}$ Genetron Health Co., Ltd, Beijing, China. ${ }^{5}$ Department of Gastrointestinal Surgery, Key laboratory of Carcinogenesis and Translational Research (Ministry of Education), Peking University Cancer Hospital \& Institute, No.52 Fucheng Road, Haidian District, Beijing 100142, China.

Received: 10 August 2017 Accepted: 15 May 2018

Published online: 22 June 2018

\section{References}

1. Torre LA, Bray F, Siegel RL, Ferlay J, Lortet-Tieulent J, Jemal A. Global cancer statistics, 2012. CA Cancer J Clin. 2015;65(2):87-108.

2. Lee SC, Guo JY, Lim R, Soo R, Koay E, Salto-Tellez M, Leong A, Goh BC. Clinical and molecular characteristics of hereditary non-polyposis colorectal cancer families in Southeast Asia. Clin Genet. 2005:68(2):137-45.

3. DA SF, Wernhoff P, Dominguez-Barrera C, Dominguez-Valentin M. Update on hereditary colorectal Cancer. Anticancer Res. 2016;36(9):4399-405.

4. Kohlmann W, Gruber SB. Lynch Syndrome. 2004 Feb 5 [Updated 2018 Feb 1]. In: Adam MP, Ardinger HH, Pagon RA, et al., editors. GeneReviews. Seattle: University of Washington, Seattle; 1993-2018. https://www.ncbi.nlm. nih.gov/books/NBK1211/. Accessed 4 June 2018.

5. Walcott FL, Patel J, Lubet R, Rodriguez L, Calzone KA. Hereditary cancer syndromes as model systems for chemopreventive agent development. Semin Oncol. 2016:43(1):134-45.

6. Huang DS, Tao HQ, He XJ, Long M, Yu S, Xia YJ, Wei Z, Xiong Z, Jones S, He $Y$, Yan $\mathrm{H}$, Wang $X$. Prevalence of deleterious ATM germline mutations in gastric cancer patients. Oncotarget. 2015;6(38):40953-8.

7. Chen W, Zheng R, Baade PD, Zhang S, Zeng H, Bray F, Jemal A, Yu XQ, He J. Cancer statistics in China, 2015. CA Cancer J Clin. 2016;66(2):115-32.

8. Rohlin A, Rambech E, Kvist A, Torngren T, Eiengard F, Lundstam U, Zagoras T, Gebre-Medhin S, Borg A, Bjork J, et al. Expanding the genotypephenotype spectrum in hereditary colorectal cancer by gene panel testing. Familial Cancer. 2016;16(2):195-203. PMID: 27696107. https://www.ncbi.nlm. nih.gov/pmc/articles/PMC5357488/.

9. Mcllhatton MA, Tyler J, Kerepesi LA, Bocker-Edmonston T, Kucherlapati MH, Edelmann W, Kucherlapati R, Kopelovich L, Fishel R. Aspirin and low-dose nitric oxide-donating aspirin increase life span in a lynch syndrome mouse model. Cancer Prev Res (Phila). 2011:4(5):684-93. 
10. Ricciardiello L, Ahnen DJ, Lynch PM. Chemoprevention of hereditary colon cancers: time for new strategies. Nat Rev Gastroenterol Hepatol. 2016;13(6):352-61.

11. Burn J, Gerdes AM, Macrae F, Mecklin JP, Moeslein G, Olschwang S, Eccles $D$, Evans DG, Maher ER, Bertario L, et al. Long-term effect of aspirin on cancer risk in carriers of hereditary colorectal cancer: an analysis from the CAPP2 randomised controlled trial. Lancet. 2011;378(9809):2081-7.

12. Sargent DJ, Marsoni S, Monges G, Thibodeau SN, Labianca R, Hamilton SR, French AJ, Kabat B, Foster NR, Torri V, et al. Defective mismatch repair as a predictive marker for lack of efficacy of fluorouracil-based adjuvant therapy in colon cancer. J Clin Oncol. 2010;28(20):3219-26.

13. Le DT, Uram JN, Wang H, Bartlett BR, Kemberling H, Eyring AD, Skora AD, Luber BS, Azad NS, Laheru D, et al. PD-1 blockade in tumors with mismatchrepair deficiency. N Engl J Med. 2015;372(26):2509-20.

14. National Comprehensive Cancer Network: ( NCCN) Clinical Practice Guidelines in Oncology Colon Cancer, Version 2. In., vol. 2016; 2016.

Ready to submit your research? Choose BMC and benefit from:

- fast, convenient online submission

- thorough peer review by experienced researchers in your field

- rapid publication on acceptance

- support for research data, including large and complex data types

- gold Open Access which fosters wider collaboration and increased citations

- maximum visibility for your research: over $100 \mathrm{M}$ website views per year 